\title{
Über das $\alpha \beta$-Dipiperidyl
}

von

\author{
Dr. Fritz Blau.
}

Aus dem I. chemischen Universitätslaboratorium in Wien.

(Vorgelegt in der Sitzung am 7. April 1892.)

Eine vorläufige Mittheilung ${ }^{1}$ über diesen Gegenstand, die ich vor ungefähr einem Jahre veröffentlichte; kann ich jetzt nach eingehenderem Studium des citirten Körpers und einiger seiner Derivate erweitern und in einigen Punkten auch corrigiren.

Die beiden von Skraup dargestellten Dipyridyle sind nach ihrer Darstellung aus den beiden Phenylendiaminen, die durch die Glycerinsynthese in Phenanthroline und weiters durch Oxydation in Dipyridyldicarbonsäuren verwandelt wurden, welche ihrerseits durch Kohlensäure-Abspaltung in die beiden isomeren Dipyridyle übergehen, ihrer Constitution nach völlig sichergestellt. Sie boten bisher ein besonderes Interesse, da man annahm und wohl noch grösstentheils annimmt, dass sie zum Nicotin in naher Beziehung stehen, insoferne als das Nicotin von der Mehrzahl der Chemiker als eines der zahlreichen denkbaren Hydrirungsproducte eines der drei $\beta$-Dipyridyle aufgefasst wird.

Die Hauptstütze dieser Ansicht bildete die Darstellung des "Isodipyridin « $\mathrm{C}_{10} \mathrm{H}_{20} \mathrm{~N}_{2}$, die von Cahours und Etard ${ }^{2}$ durch Einwirkung von Ferricyankalium auf Nicotin und auch auf anderem Wege vollführt wurde. Es muss aber dazu bemerkt werden, dass man durch nichts verleitet wird, eine thatsächliche Beziehung zwischen einem Dipyridyl und dem sogenannten

1 Ber. d. deutschen chem. Gesellschaft, XXIV, S. 326.

2 Bul. soc. chim. (2) 34, p, 452 . 
Isodipyridin der genannten Forscher anzunehmen als blos durch die Namen.

Ferner wurden zwei Basen von der Formel $\mathrm{C}_{\mathbf{1 0}} \mathrm{H}_{\mathbf{1 4}} \mathrm{N}_{2}$ dargestellt, das Isonicotin von Weidel und Russo ${ }^{1}$ und das Nicotidin von Skraup und Vortmann, ${ }^{2}$ deren Eigenschaften zwar durchaus nicht gegen eine Ortsisomerie mit dem Nicotin sprechen, aber auch keineswegs zu einer solchen Annahme Veranlassung geben.

Alles, was man streng genommen über die Constitution des Nicotins sagen kann, ist folgendes:

1. Es ist $z w$ eifellos eine ditertiäre Base. Dafür spricht das Verhalten gegen Jodalkyle, sowie der Umstand, dass es weder mit Säurechloriden reagirt (trotz einiger gegentheiliger Behauptungen), noch eine Nitrosoverbindung liefert oder sich mit Schwefelkohlenstoff verbindet.

2. Es enthält keine an Stickstoff gebundenen Alkylgruppen.

3. Es enthält einen Pyridinkern, der bei der Oxydation erhalten bleibt und $\beta$-Pyridincarbonsäure liefert.

DieserKern ist höchsten s vierfach hydrirt (wegen der tertiären Natur der Base), er ist aber höchst wahrscheinlich frei von addirten Wasserstoffen, da die ganz ausgezeichnete Ausbeute an Pyridincarbonsäure bei der Oxydation eines Hydropyridinderivats ganz vereinzelt dastehen würde.

Alle Vermuthungen, welchè darüber hinausgehen, sind durch nichts Experimentelles gestützt.

Liebrecht, der von der Voraussetzung ausgeht, dass im Nicotin ein Dipyridylderivat vorliege, hat dasselbe mit Natrium und Alkohol nach der von Ladenburg angegebenen Methode erschöpfend hydrirt und eine Base von der Formel $\mathrm{C}_{10} \mathrm{H}_{20} \mathrm{~N}_{2}$ erhalten, von der er zwar unentschieden lässt, welches der drei möglichen $\beta$-Dipiperidyle vorliege, die er aber mit Bestimmtheit als ein Dipiperidyl bezeichnet.

1. Monatsh. f. Chem., III, S. 850.

2 Ebenda, IV, S. 597. 
Er liefert jedoch dafür absolut keinen Beweis, so dass man correct nur von einem Hexahydronicotin, nicht aber von einem Dipiperidyl sprechen kann, und wenn er sagt, man könne die Constitution des dem Nicotin zu Grunde liegenden Dipyridyls dadurch ermitteln, dass man das totale Reductionsproduct (Hexahydronicotin) mit den aus Dipyridylen bekannter Constitution durch erschöpfende Reduction erhaltbaren Dipiperidylen vergleiche und ermittle, mit welchem derselben das Hexahydronicotin identisch sei, so ist dies correct dahin $z u$ modificiren, dass man auf diesem Wege ermitteln werde, ob überhaupt ein Dipyridyl dem Nicotin zu Grunde liege.

Als ich mich der ganz besonders mühsamen Arbeit der Darstellung einer genügenden Menge von $\alpha \beta$-Dipyridyl unter$z o g$, erschien mir die Annahme, dass das Nicotin ein Dipyridylderivat sei, zwar nicht hervorragend wahrscheinlich, aber doch immerhin ganz plausibel, und speciell das $\alpha \beta$-Dipyridyl war zunächst in Betracht zu ziehen, da im Nicotin eine optisch active Substanz vorliegt, deren Drehungsvermögen nach Liebrecht im Hexahydronicotin erhalten bleibt, so dass auch in diesem mindestens ein Kohlenstoffatom vorhanden sein muss, das Veranlassung zur Activität bildet.

Dieses kann natürlich nur eines der beiden Kohlenstoffatome sein, an welchen die beiden Kerne verknüpft sind, und wenn man die beiden Formeln.<smiles>CCC(C)C1CCCNC1</smiles>

$(\beta \beta)$-Dipiperidyl

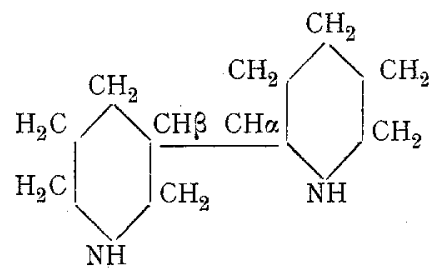

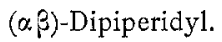

betrachtet, so sieht man wohl, dass das letztere alle Eigenschaften einer Substanz hat, die optisch activ auftreten kann, während die allerdings vorhandene Asymmetrie des ersteren vermuthlich zum Zustandekommen optischer Activität nicht ausreichen dürfte. ${ }^{1}$

${ }^{1}$ Ich will durchaus nicht behaupten, dass ein Körper, wie das $\beta \beta$-Dipiperidyl, nicht doch optisch activ sein könne, ich halte dies nur für viel weniger wahrscheinlich als bei einem $\sigma$ Derivat. 
Ausserdem veranlasste mich noch ein Umstand, zunächst das ( $\alpha \beta$ )-Dipyridyl in Untersuchung $z u$ ziehen (übrigens ein Umstand, der überhaupt gegen die Dipyridylstructur des Nicotins spricht), nämlich der, dass das ( $\alpha \alpha)$-Dipiperidyl (welches ich vor längerer Zeit aus Picolinsäure dargestellt habe) ${ }^{1}$ einen höheren Siedepunkt besitzt, als ihn Li e bre cht für sein Hexahydronicotin ( $\beta x$-Dipiperidyl angibt.

Es war daher ein Hauptgewicht auf die genaue Feststellung des Siedepunktes des $\alpha \beta$-Dipiperidyls zu legen, um zu constatiren, ob hier nicht doch vielleicht eine Ausnahme von der sonst giltigen Regel, dass $\alpha$-Substitutionsproducte am niedrigsten sieden, stattfinde.

Was die Darstellung des Ausgangsmaterials betrifft, so will ich hier bemerken, dass die Ausbeute, die ich an Phenanthrolin erhielt, um etwa ein Drittel hinter der von Skraup angegebenen zurückblieb, dass ebenso die Oxydation $z \mathrm{u}$ Phenanthrolinsäure schlechtere Resultate ergab, dass hingegen die Ausbeute an Dipyridyl aus der Säure besser war, als ich erwarten konnte, nämlich $50 \%$ der Theorie an reiner Base.

Die Angaben Skraup's über diese haoe ich in Allem bestätigt gefunden, nur den Siedepunkt fand ich bei 295.5 bis $296 \cdot 5^{\circ}$ corr. Skraup's Angaben sind zweifellos uncorrigirt $z u$ nehmen.

Die Reduction des Dipyridyls wurde mit Natrium und Alkohol in bekannter Weise vorgenommen und mehrfach wiederholt; es wurde auch mit Amylalkohol und Natrium gearbeitet, das Resultat ist aber immer dasselbe. Ein gewisses Quantum der Substanz wird nur theilweise hydrirt, ein anderes gibt höher siedende, etwas schmierige Producte, die bei der Gewinnung der Base durch Ausschütteln mit Äther und Fractioniren nicht völlig entfernt werden konnten.

(Da jene Substanz, welche ich zur Zeit meiner vorläufigen Mittheilung in der Hand hatte, nur auf diese Weise gereinigt war und daher kleine Mengen anderer Substanzen enthalten haben dürfte, muss ich einige Schmelzpunktsangaben modificiren.)

1 Monatshefte X, S. 294. 

schlagen.

Zur Reindarstellung habe ich den folgenden Weg einge-

Die Lösung von Natriumäthylat, welche die Base enthält, wird mit Wasser zersetzt, mit Salzsäure angesäuert und nach dem Eindampfen mit Kali übersättigt und mit Wasserdämpfen übergetrieben. (Dies dauert ziemlich lang.) Es bleibt ein wenig eines alkalischen Öles in derRetorte, das ich nicht näher untersuchen konnte, welches offenbar die Hauptverunreinigung des rohen Dipiperidyls bildete.

Wenn man die Destillate mit Salzsäure eindampft, so bleibt eine nicht sehr hygroskopische Krystallmasse zurück, welche in möglichst wenig heissem Wasser gelöst, auf Zusatz von Alkohol im Überschuss ausfällt; aus der alkoholischen Mutterlauge fällt durch Ätherzusatz eine weitere Menge.

Aus den eingedampften letzten Mutterlaugen kann durch abermaliges Destilliren mit Wasserdämpfen aus . kalischer Lösung ein Destillat erhalten werden, aus dem in der schon beschriebenen Weise noch eine kleine Quantität des Chlorhydrats gewonnen werden kann.

Dieses Chlorhydrat ist völlig luftbeständig, seine wässerige Lösung hinterlässt nach dem Verdunsten dasselbe sofort in festem Zustande.

Die freie Base wird gewonnen durch Zerlegen der concentrirten wässerigen Lösung des Salzes mit Überschuss von Kali; das abgeschiedene Ö1 ${ }^{1}$ wird mit Äther aufgenommen. Wird dieser nach dem Trocknen verdunstet, so bleibt ein bald grösstentheils erstarrendes Öl zurück, welches im Fractionirkölbchen im Wasserstoffstrom bei langsam bis in die Nähe des Siedepunktes erhöhter Temperatur getrocknet und destillirt bei $267-268^{\circ}$ corr. übergeht; das völlig erstarrende Destillat zeigt noch keinen constanten Schmelzpunkt (es beginnt bei $40^{\circ} \mathrm{zu}$ schmelzen und ist bei $60^{\circ}$ völlig verflüssigt).

Die Reinigung wird am besten durch Wiederholung der ganzen eben geschilderten Operation vorgenommen, wodurch die Base als ein fester Körper von schon angegebenem Siedepunkt und vom Schmelzpunkt $68-69^{\circ}$ erhalten wird.

\footnotetext{
1 Erstarrt oft schon bei der Ausscheidung.
} 
Die reine Base ist ein sehr hygroskopischer, energisch Kohlensäure anziehender, stark alkalischer Körper; nur ganz kurze Zeit mit der Atmosphäre in Berührung nimmt sie Wasser und Kohlensäure auf, so dass der Schmelzpunkt sofort ausserordentlich herabgedrückt wird, daher stammt auch meine erste ${ }^{1}$ viel zu niedrige Schmelzpunktsangabe, und ich halte es nicht für völlig ausgeschlossen, dass selbst der eben angeführte Schmelzpunkt $68-69^{\circ}$ noch ein wenig zu niedrig gegriffen sei: Die Substanz ist eben ausserordentlich empfindlich.

Dass thatsächlich das 12 fache Hydrirungsproduct des Dipyridyls, ein Dipiperidyl, vorliegt, wird durch die Analyse erwiesen.

I. $0 \cdot 2337 \mathrm{~g}$ Substanz gaben $0 \cdot 2461 \mathrm{~g}$ Wasser und $0 \cdot 6121 \mathrm{~g}$ Kohlensäure.

II. $0 \cdot 1954 \mathrm{~g}$ Substanz gaben $0 \cdot 2108 \mathrm{~g}$ Wasser und $0 \cdot 5129 \mathrm{~g}$ Kohlensäure.

III. 0.1661 $g$ Substanz gaben bei einem Barometerstand von $754 \mathrm{~mm}$ und einer Temperatur von $18^{\circ} 5.24 \cdot 3 \mathrm{~cm}^{3}$ feuchten Stickstoff.

In 100 Theilen:

\begin{tabular}{|c|c|c|c|}
\hline \multicolumn{3}{|c|}{ Gefunden } & \multirow{2}{*}{$\begin{array}{l}\text { Berechnet für } \\
\mathrm{C}_{10} \mathrm{H}_{20} \mathrm{~N}_{2}\end{array}$} \\
\hline I. & II. & III. & \\
\hline$\ldots 71 \cdot 43$ & $71 \cdot 54$ & - & $71 \cdot 37$ \\
\hline$\ldots 11 \cdot 72$ & $12 \cdot 00$ & - & $11 \cdot 91$ \\
\hline$\ldots-$ & - & $16 \cdot 72$ & $16 \cdot 71$ \\
\hline
\end{tabular}

Das Dipiperidyl löst sich äusserst leicht in Wasser, mit viel Wasser entsteht eine Trübung, sehr leicht in Alkohol, ziemlich schwer in kaltem, viel reichlicher in siedendem Äther.

(Letzteres Verhalten kann auch zur Reinigung der Base benutzt werden, doch ist das Absaugen der so hygroskopischen Substanz ziemlich unangenehm.)

Es ist ferner in den meisten gebräuchlichen Lösungsmitteln leicht löslich.

Die Salze mit starken Säuren reagiren völlig neutral, und lässt sich daher die Base mit Salzsäure alkalimetrisch titriren;

1 Vorl. Mittheilung loco citato. 
der so gefundene Stickstoffgehalt, ein $\overline{\mathrm{H}} \mathrm{Cl}$ auf ein $\mathrm{N}$ gerechnet, stimmt mit dem durch Elementaranalyse gefundenen überein.

Das schon öfter erwähnte

\section{Chlorhydrat}

wird am besten aus einer alkoholischen Lösung der Base durch concentrirte Salzsäure gefällt; es ist durchaus nicht hygroskopisch, aber äusserst leicht löslich in Wasser, schwer in Alkohol, nicht in Äther.

Beim Erhitzen bis über $300^{\circ}$ schmilzt es nicht und sublimirt noch höher erhitzt fast unzersetzt.

1. $0 \cdot 2473 g$ Substanz gaben $0 \cdot 2008 g$ Wasser und $0 \cdot 4490 g$ Kohlensäure.

2. $0 \cdot 2374 g \cdot$ Substanz gaben $0 \cdot 2806 g$ Chlorsilber, entsprechend $0.0694 \mathrm{~g}$ Chlor.

In 100 Theilen:

\begin{tabular}{|c|c|c|}
\hline \multicolumn{2}{|c|}{ Gefunden } & Berechnet für \\
\hline I. & II. & $\mathrm{C}_{10} \mathrm{H}_{20} \mathrm{~N}_{2.2}$ \\
\hline C . . $49 \cdot 52$ & - & $49 \cdot 78$ \\
\hline $\mathrm{H} \ldots 9 \cdot 05$ &.- & $9 \cdot 15$ \\
\hline $\mathrm{Cl} \ldots-$ & $29 \cdot 23$ & $29 \cdot 38$ \\
\hline
\end{tabular}

Wird zu einer Lösung des Chlorhydrats in Wasser Platinchlorid gesetzt, so krystallisirt bei nicht zu geringer Concentration ein Platindoppelsalz von orangegelber Farbe, leicht löslich in Wasser, vom Schmelzpunkt 237-238, der sich durch Umkrystallisiren aus wenig heissem, mit einer Spur Salzsäure versetztem Wasser nicht mehr ändert.

Das Chloroplatinat enthält zwei Moleküle Krystallwasser, die bei $100^{\circ}$ entweichen.

$0 \cdot 1493 g$ Substanz verloren beim Trocknen $0 \cdot 0084 \mathrm{~g}$ Wasser.

In 100 Theilen:

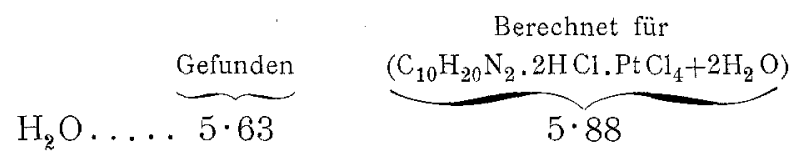

Der Rückstand vom Gewichte $0 \cdot 1409 \mathrm{~g}$ hinterliess beim Glühen $0.0477 g$ Platin. 
In 100 Theilen:

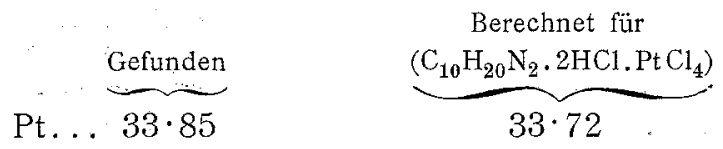

Auf Zusatz von Goldchlorid zur ziemlich verdünnten Lösung des Chlorhydrats fallen hellgelbe Kryställchen des Doppelsalzes $\left(\mathrm{C}_{\mathbf{1 0}} \mathrm{H}_{\mathbf{2 0}} \mathrm{N}_{2} \cdot 2 \mathrm{HCl} \cdot 2 \mathrm{AuCl}_{3}\right)$. Der Schmelzpunkt liegt bei $211-212^{\circ}$. Aus den Mutterlaugen krystallisirt dieselbe Verbindung.

$0.1673 \mathrm{~g}$ Substanz hinterliessen beim Glühen $0.0778 \mathrm{~g}$ Gold. In 100 Theilen:



Silbernitrat, zur Lösung der freien Base in Wasser gesetzt, erzeugt einen geringen Niederschlag, der sich beim Kochen schwärzt.

Quecksilberchlorid fällt die freie Base; die Fällung ist löslich im Überschuss des Dipiperidyls.

Beim Kochen löst sich der Niederschlag und erscheint beim Abkühlen sofort wieder.

Er ist nicht constant zusammengesetzt.

Aus einer selbst sehr concentrirten Lösung des Chlorhydrats wird durch Quecksilberchlorid nichts ausgeschieden.

Auf Zusatz einer heissen alkoholischen Lösung von überschüssiger Pikrinsäure zur ebenfalls heissen Lösung von Dipiperidyl fällt ein Öl, das beim Reiben erstarrt. Der Schmelzpunkt dieses Pikrats liegt bei $210^{\circ}$, nach einmaligem Umkrystallisiren aus heissem Alkohol bei $215^{\circ}$ und ändert sich dann nicht weiter.

Eine sehr verdünnte heisse Lösung von Dipiperidyl in Alkohol scheidet auf Zusatz von Pikrinsäure zunächst nichts und nach längerem Stehen dasselbe Salz krystallisirt aus. Der Schmelzpunkt wurde direct zu $215^{\circ}$ gefunden.

$0.2388 \mathrm{~g}$ Substanzen gaben $0.0928 \mathrm{~g}$ Wasser und $0.3711 \mathrm{~g}$ Kohlensäure. 
In 100 Theilen:

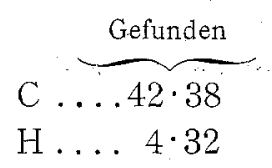

Berechnet für

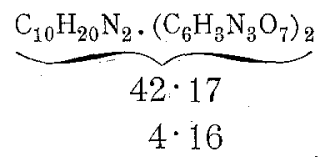

Das $\alpha \beta$-Dipiperidyl enthält zwei secundäre Stickstoffatome; dementsprechend war zu erwarten, dass es sich mit Säurechloriden zu Säureamiden verbinden wird, dass es ferner Schwefelkohlenstoff addire unter Bildung von dipiperidodithiocarbaminsaurem Dipiperidyl, und dass es mit salpetriger Säure eine Nitrosoverbindung liefere, welche zwei (NO)-Gruppen enthält.

Wird $\alpha \beta$-Dipiperidyl in wässeriger Lösung mit Benzoylchlorid und- Kalilauge geschüttelt, nach Vollendung der Reaction mit Äther aufgenommen und dieser grösstentheils verdunstet, so krystallisirt nach einigem Stehen die Benzoylverbindung aus. Ich begnügte mich mit dem qualitativen Nachweis der Abwesenheit von Chlor und Anwesenheit von Stickstoff.

In gleicher Weise wird unter Anwendung von Benzolsulfochlorid nach Verdunsten eines Theiles des Äthers das Benzolsulfodipiperid in Krystallen erhalten, welche fast farblos, in Äther ziemlich schwer löslich sind und bei $157^{\circ}$ schmelzen.

Auch hier begnügte ich mich mit der Constatirung, dass das Product chlorfrei, aber stickstoff- und schwefelhältig sei.

\section{Nitrosodipiperidy 1.}

Wird eine schwach schwefelsaure Lösung der Base mit einer neutralen Kalium- oder Natriumnitritlösung (dem $1 \frac{1}{2}$ bis 2 fachen der Theorie) versetzt, so scheidet sich nach einiger Zeit ein Öl ab, das Neigung zum Krystallisiren zeigt, aber nur theilweise erstarrt. Wird nach mehrstündigem Stehen mit alkoholhaltigem Äther ausgeschüttelt und dieser nach dem Trocknen fast verdunstet, so krystallisirt nach einiger Zeit aus dem alkoholischen Rückstand die Nitrosoverbindung, die abgesaugt und aus Alkohol umkrystallisirt wird; aus den vereinigten Mutterlaugen kann nach dem Abdunsten zur völligen 
Trockniss durch Befeuchten mit absolutem Alkohol und Zusatz von trockenem Äther nach Einwerfen eines Kryställchens der reinen Verbindung eine weitere Menge gewonnen werden. Der Schmelzpunkt von $87 \cdot 5-88 \cdot 5^{\circ}$ wird durch Umkrystallisiren nicht geändert.

Die Substanz zeigt den charakteristischen Geruch der Nitrosamine, ist in Wasser sehr schwer löslich; Zusatz von Säuren erhöht die Löslichkeit, doch wird auch aus einer stark mit Salzsäure versetzten Lösung die Base durch Äther aufgenommen, ein Beweis, dass beide Stickstoffatome in Reaction getreten sein müssen und also beide secundärer Natur sind, was übrigens durch die Analyse vollends ausser Zweifel gesetzt wird.

$0.1548 \mathrm{~g}$ Substanz gaben bei einem Barometerstande von $744 \mathrm{~mm}$ und einer Temperatur von $21^{\circ} 34.7 \mathrm{~cm}^{3}$ feuchten Stickstoff.

In 100 Theilen:

$\mathrm{N} \ldots \underbrace{\text { Gefunden }}_{24 \cdot 95} \quad \frac{\mathrm{C}_{10} \mathrm{H}_{18} \mathrm{~N}_{2}(\mathrm{NO})_{2}}{24 \cdot 83}$

Duirch längeres Erwärmen der Nitrosobase mit concentrirter Salzsäure wird die ursprüngliche Base theilweise regenerirt.

Wird ein wenig Dipiperidyl auf einem Uhrglas geschmolzen und nach dem Abkühlen mit Schwefelkohlenstoff befeuchtet, so tritt Vereinigung zu einer hellgelblichweissen Masse ein, die aber in Folge der Heftigkeit der Reaction grösstentheils weggeschleudert wird.

Zur Darstellung der Verbindung wird am besten eine alkoholische Lösung der Base mit reinem, mit A1kohol verdünntem Schwefelkohlenstoff versetzt; sofort füllt das Reactionsproduct in deutlichen Krystallen.

Nach einigem Stehen wird von der Lauge getrennt und aus heissem Alkohol umkrystallisirt. Rasches Arbeiten ist erforderlich, da die Verbindung leich verharzt.

Sie zeigt den Schmelzpunkt $205^{\circ}$ unter Zersetzung. 
Aus ätherischer Lösung wird Dipiperidyl durch Schwefelkohlenstoff sofort und vollständig gefällt, selbst bei sehr grosser Verdünnung, und zwar in sehr feinen Krystallen, die beim Absaugen an feuchter Luft grösstentheils verharzen. "Die abgesaugte Masse zeigt eine ausserordentliche Neigung, bei leiser Berührung elektrisch zu werden, so dass es nicht möglich ist, sie in gepulvertem Zustande ohne bedeutenden Verlust aus einem Gefäss in ein anderes zu bringen.

Aus Alkohol werden weniger feine Krystalle erhalten, die nicht so stark elektrisch werden und nicht so leicht verharzen, wie die aus Äther, aber doch auch unbeständig sind und sich an feuchter Luft rasch verändern.

Darum wurde auch bei der Analyse der Schwefelgehalt viel zu niedrig gefunden, wenn auch unzweideutig auf eine Verbindung von je 1 Molekül $\mathrm{CS}_{2}$ mit 1 Molekül Dipiperidyl stimmend.

Zwei Schwefelbestimmungen ergaben $22 \cdot 5$ und $24 \% \mathrm{~S}$, während die Verbindung $\left(\mathrm{C}_{10} \mathrm{H}_{20} \mathrm{~N}_{2}\right) \mathrm{CS}_{2} 26 \cdot 2$, die Verbindung $2\left(\mathrm{C}_{10} \mathrm{H}_{20} \mathrm{~N}_{2}\right) \mathrm{CS}_{2} 15 \cdot 3 \%$ erfordert.

Das Dipiperidyl vereinigt sich mit Jodmethyl.

Eine Untersuchung des Additionsproductes erschien bei der geringen mir noch zu Gebote stehenden Menge der Base aussichtslos, da ja bekanntlich secundäre Basen mit Jodalkylen nicht glatt reagiren.

Mein $\alpha \beta$-Dipiperidyl ist mit dem von Liebrecht erhaltenen Hexahydronicotin $\mathrm{n}$ icht identisch, wie sich dies aus dem Vergleich seiner Eigenschaften mit den des Liebrecht'schen Körper zweifellos ergibt.

$$
\text { Base } \overbrace{\begin{array}{l}
\text { Smp. . .68-69-69 } \\
\text { Sp. . . . 268-269 }
\end{array}}^{\alpha \beta \text {-Dipiperidyl }}
$$

Chlorhydrat

Platinat

Aurat

Nitrosoverbindung
Luftbeständig Smp. $238^{\circ}$ Smp. $212^{\circ}$ Smp. $88^{\circ}$
Hexahydronicotin

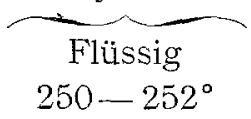

Zerfliesslich

Smp. $202^{\circ}$

Smp. $131^{\circ}$

Ö1.

Der Umstand, dass das Hexahydronicotin optisch activ ist, während meine Base, die synthetisch gewonnen wurde, 
natürlich inactiv ist, würde zwar kleine Differenzen in den Eigenschaften erklären, nicht aber solche von der angeführten Grössenordnung.

Da das aß-Dipiperidyl, wie a priori wahrscheinlich, thatsächlich höher siedet, als das a.-Dipiperidyl (Sp. 259), da ferner anzunehmen ist, dass dieses Ansteigen beim Übergang zum $\beta \beta$ - und $\beta \gamma$-Dipiperidyl bestehen bleiben wird, dass aber zum mindesten kein Herabsinken des Siedepunktes dieser beiden Dipiperidyle unter den des $\beta \alpha$-oder gar $\alpha \alpha$-Dipiperidyl (Sp. $269^{\circ}$, resp. $259^{\circ}$ ) statthaben wird, während Liebrecht den Siedepunkt seines Hexahydronicotins zu $250-252^{\circ}$ angibt, so erscheint es äusserst unwahrscheinlich, dass in Liebrecht's Körper überhaupt ein Dipiperidyl vorliegt, und damit auch jede Beziehung eines Dipyridyls zum Nicotin von derselben Unwahrscheinlichkeit.

Diese Schlüsse habe ich seinerzeit in meiner schon citirten vorläufigen Mittheilung gezogen.

Dass ich sie nunmehr nicht vollauf festhalten kann, liegt nicht an einer Änderung meiner Ansichten, sondern daran, dass die Resultate meiner seit einiger Zeit aufgenommenen Untersuchung des Liebrecht'schen Körpers mit seinen Angaben durchaus nicht in Einklang zu bringen sind.

Ich habe zur Hydrirung drei Präparate verwendet. Ein Nicotin, dâs sich seit längerer Zeit im Laboratorium vorfand, mir unbekannten Ursprungs, die beiden anderen zu verschiedenenZeiten von Merk in Darmstadt bezogen. (DieselbeQuelle gibt Liebrecht an.)

Die Präparate hatten nach leichter Reinigung den Siedepunkt $246-247^{\circ}$ (corr.) und stimmten in jederBeziehung unter sich, sowie mit den bekannten Angaben über das Nicotin überein.

Die Reduction wurde, genau wie Liebrecht es angibt, vorgenommen, aber in allen Fällen nicht mit dem erwarteten Resultate.

Ich erhielt keinen einheitlichen, bei $250-252^{\circ}$ siedenden Körper, sondern offenbar ein Gemisch, das von 235-265 überging, aber auch da noch einen nicht unbedeutenden Rückstand hinterliess. 
Allerdings kochte die Hauptfraction bei $250-252^{\circ}$, aber beim Ausfractioniren zeigte sich deutlich die Tendenz, in einen höher und einen niedriger siedenden Antheil zu zerfallen, ohne dass es gelungen wäre, eine völlige Trennung zu erzielen.

Nur so viel war $z$ u ersehen, dass die niedrige Fraction hauptsächlich ein schwer lösliches, die höhere ein leicht lösliches (niedrig schmelzendes) Chloroplatinat lieferte.

Ich habe daher alle Fractionen vereinigt noch mehrmals der Einwirkung von Natrium und Alkohol unterzogen; das Resultat war dasselbe Gemisch. Auch mit Amylalkohol wurde nichts Anderes erzielt.

Ich trieb nun die Base mit Wasserdämpfen über. In der Retorte blieb ein nicht sehr bedeutender, nicht flüchtiger Rückstand, mit dessen Untersuchung ich noch beschäftigt bin.

Das Destillat wurde mit Salzsäure eingedampft, der nicht krystallisirende Syrup mit Kali zerlegt, mit Äther aufgenommen und nach dem Trocknen in bekannter Weise destillirt. Das Product war unverändert geblieben, nur blieb jetzt über $265^{\circ}$ kaum ein Rückstand im Fractionirkolben.

Die einzelnen Fractionen wurden nun mit Salzsäure neutralisirt, mit Platinchlorid gefällt und systematisch umkrystallisirt. Es wurde ein schwer lösliches Chloroplatinat vom Schmelzpunkt $218^{\circ}$ erhalten, das, wie sich bald zeigte, rein war, und ein äusserst leicht lösliches; ob in den Zwischenfractionen noch ein drittes Salz sich befand, oder nur ein Gemisch vorlag, muss ich zunächst unentschieden lassen.

Durch Zerlegung der Fraction von Smp. $218^{\circ}$ mit Schwefelwasserstoff und Ausfällung der Base aus der eingedickten Chlorhydratlösung mit Kali im Überschuss erhielt ich eine nunmehr constant bei $237-239^{\circ},\left(243-245^{\circ}\right.$ corr. $)$ übergehende Flüssigkeit, die dasselbe Chloroplatinat lieferte, aus dem sie hergestellt war (Smp. unverändert $218^{\circ}$ ), dessen Platingehalt aber nur $32 \cdot 6 \%$ betrug, während die Formel $\left(\mathrm{C}_{10} \mathrm{H}_{20} \mathrm{~N}_{2} .2 \mathrm{HCl} . \mathrm{PtCl}_{4}\right)$ $33 \cdot 72 \%$ verlangt.

Das Aurat schmolz bei $187^{\circ}$.

Die freie Base gab bei der Analyse Zahlen $\mathrm{C}=72 \cdot 3 \%$, $\mathrm{H}=11 \cdot 8 \%, \mathrm{~N}=15 \cdot 8 \%$, die nicht auf die Formel $\mathrm{C}_{10} \mathrm{H}_{20} \mathrm{~N}_{2}$ wohl aber auf $\mathrm{C}_{11} \mathrm{H}_{22} \mathrm{~N}_{2}$ stimmen. 
Damit ist auch der oben erwähntePlatingehalt des Chloroplatinats in Übereinstimmung.

Da nun eine Formel $\mathrm{C}_{11} \mathrm{H}_{22} \mathrm{~N}_{2}$ bei einem Körper, der durch Wasserstoffaddition aus $\mathrm{C}_{10} \mathrm{H}_{14} \mathrm{~N}_{2}$ entsteht, absolut nicht $\mathrm{zu}$ erklären ist, untersuchte ich das Nicotin, welches mir zur Verfügung stand, auf eine Verunreinigung mit einem bisher unbekannten, möglicherWeise in der Natur vorkommenden Methylnicotin, bisher allerdings noch ohne Erfolg, doch wird die Untersuchung fortgesetzt.

Die aus dem leicht löslichen Chloroplatinat dargestellte höher siedende Base erwies sich als noch unrein; ich kann hier nur so viel sagen, dass sie mit dem synthetischen $\alpha \beta$-Dipiperidyl ebensowenig identisch ist, als die aus dem schwer löslichen Chloroplatinat erhaltene, dass aber auch ihre Eigenschaften mit den Angaben Liebrecht's nicht in Einklang zu bringen sind.

Die Untersuchung der Hydrirungsproducte des Nicotins, mit grösseren Materialmengen fortgesetzt, wird jedenfalls zur Klarheit führen und hoffe ich darüber bald nähere Aufschlüsse bringen zu können. 